\title{
Updated Efficacy and Safety Outcomes for Patients with Well-Differentiated Pancreatic Neuroendocrine Tumors Treated with Sunitinib
}

\author{
Nicola Fazio ${ }^{1} \cdot$ Matthew Kulke ${ }^{2} \cdot$ Brad Rosbrook $^{3} \cdot$ Kathrine Fernandez $^{4} \cdot$ Eric Raymond $^{5}$
}

Accepted: 5 December 2020 / Published online: 7 January 2021

(c) The Author(s) 2021

\begin{abstract}
Background Sunitinib prolonged progression-free survival (PFS) versus placebo in patients with metastatic pancreatic neuroendocrine tumors (panNETs) in a phase III trial. The efficacy and safety of sunitinib in patients with panNETs were confirmed in an open-label phase IV trial.

Objective To assess the clinical benefit with sunitinib using the combined data from these trials.

Patients and methods An updated overall survival (OS) in patients with panNETs for the phase IV trial was provided, and an analysis of results from the sunitinib-treated combined cohort from the phase III and IV trials (combined cohort) was conducted to assess PFS, OS, and objective response rate (ORR).

Results The updated median OS for the phase IV trial was 54.1 months (95\% CI 37.9-not reached). Investigator-assessed median PFS for the combined cohort $(n=102)$ was 12.9 months (95\% CI 7.4-16.7) with a significant benefit versus placebo in the phase III trial $(n=35)$ (HR $0.429 ; 95 \%$ CI $0.245-0.752 ; p=0.001)$. Median OS could not be calculated for the combined cohort or placebo group due to the high number of patients censored; however, the estimated HR of 0.303 (CI $0.100-0.921 ; p=0.013$ ) favored sunitinib. ORR for the combined cohort was $16.7 \%$ (95\% CI 10.0-25.3). Sunitinib was well tolerated in both trials with a safety profile similar to previously seen in other studies.

Conclusions The combined analysis of these studies confirms the objective tumor responses and improvements in PFS observed in the initial phase III trial, providing further support for the clinical benefit of sunitinib in patients with advanced panNETs.
\end{abstract}

ClinicalTrials.gov Identifiers NCT00428597 and NCT01525550.

Nicola Fazio

nicola.fazio@ieo.it

1 Division of Gastrointestinal Medical Oncology and Neuroendocrine Tumors, European Institute of Oncology, IEO, IRCCS, Via Ripamonti 435, 20141 Milan, Italy

2 Boston University and Boston Medical Center, Boston, MA, USA

3 Pfizer Inc, La Jolla, CA, USA

4 Pfizer Inc, Cambridge, MA, USA

5 Department of Medical Oncology, Paris Saint-Joseph Hospital Group, Paris, France

\section{Key Points}

The combined data from the phase III placebo-controlled and phase IV trials in patients with pancreatic neuroendocrine tumors (panNETs) confirmed and strengthened efficacy and safety of sunitinib.

The objective tumor response rate $(16.7 \%)$ was encouraging in the combined sunitinib-treated cohort.

Objective tumor response rate may be a sunitinib-related factor to be considered in panNETs when tumor shrinkage is needed. 


\section{Introduction}

Pancreatic neuroendocrine tumors (panNETs), which arise from endocrine cells of the pancreas, are relatively rare. Surgery is the best treatment option for patients with locally advanced or oligometastatic resectable disease but for patients with unresectable advanced disease, options for treatment remain limited and prognosis is poor [1].

Dysregulation of angiogenic factors, such as vascular endothelial growth factor receptors (VEGFRs) and plateletderived growth factor receptors (PDGFRs), play a role in the growth of the highly vascularized panNETs [2,3]. Currently available treatment options for panNETs include somatostatin analogs, cytotoxic chemotherapy, peptide receptor radionuclide therapy, mammalian target of rapamycin inhibitors, and tyrosine-kinase inhibitors (TKIs) [1]. Sunitinib is the only TKI available in clinical practice for the treatment of patients with advanced/metastatic panNETs. It targets multiple receptors in angiogenesis and tumor proliferation, including VEGFR 1-3 and PDGFR-A and B [4-7]. Sunitinib was approved in 2010 in the European Union and in 2011 by the US Food and Drug Administration (FDA) for the treatment of progressive, well-differentiated panNETs in patients with unresectable locally advanced or metastatic disease [7, 8].

Sunitinib was shown to prolong progression-free survival (PFS) versus placebo in patients with metastatic, well-differentiated panNETs in a pivotal phase III clinical trial (A6181111, NCT00428597) [9]. The independent data-monitoring committee (DMC) for this trial recommended early trial termination, as early results favored sunitinib and there was an increased number of deaths and serious adverse events (AEs) in the placebo group [8]. Specifically, investigator-assessed PFS favored patients treated with sunitinib versus placebo (hazard ratio (HR) $0.42 ; 95 \%$ confidence interval (CI) $0.26-0.66 ; p<0.001$; median 11.4 (95\% CI 7.4-19.8) vs. 5.5 (95\% CI 3.6-7.4) months) [9]. In a retrospective analysis performed on radiographic data using a blinded independent central review (BICR), PFS was assessed as 12.6 (95\% CI 11.1-20.6) months and 5.8 (95\% CI 3.8-7.2) months for the sunitinib and placebo arms, respectively (HR $0.32 ; 95 \%$ CI $0.16-0.55 ; p<0.0001$ ) [10].

The efficacy and safety of sunitinib for the treatment of patients with well-differentiated advanced/metastatic panNETs were confirmed by an open-label, phase IV trial (A6181202, NCT01525550) that was conducted to meet post-approval regulatory requirements. The median PFS in this trial, as assessed by investigators, was 13.2 months (95\% CI 10.9-16.7) for all patients [11].

In light of recent reports suggesting that objective response rate (ORR) is a valuable primary endpoint for efficacy in treatments for panNETs [12-14], we performed an analysis of the combined investigator-assessed ORR data from treatment-naïve patients in the pivotal phase III and phase IV trials. ORR was previously a secondary endpoint in both trials. An analysis of the PFS, overall survival (OS), and safety data for this combined sunitinib cohort was also performed in order to provide a more complete description of the efficacy of sunitinib in the treatment of panNETs.

\section{Methods}

\subsection{Study Design}

As previously described, the phase III trial was a multinational, randomized, double-blind, placebo-controlled, phase III trial of sunitinib versus placebo in patients with progressive advanced/metastatic well-differentiated panNETs [15]. Overall, 171 patients with panNETs were enrolled in the study between 7 June 2007 and 15 April 2009. Patients were randomized $1: 1$ to receive either sunitinib $37.5 \mathrm{mg}$ once daily on a continuous daily dosing (CDD) schedule or matching placebo. The primary objective of the study was to compare PFS in the sunitinib arm versus placebo arm. Secondary objectives included OS and ORR in the sunitinib and placebo treatment arms. This study was designed to detect a $50 \%$ improvement in median PFS with a target enrollment of 340 patients. An interim analysis was planned when 130 events had occurred, and the final analysis was to be conducted when 260 events had occurred. The conduct of the study was overseen by an independent DMC.

The phase IV trial was a single-arm, multinational, multicenter, open-label trial to confirm the efficacy and safety of sunitinib in patients with progressive, advanced metastatic, well-differentiated, unresectable panNETs. Overall, 106 patients with panNETs were enrolled in the study between 6 June 2012 and 19 March 2016. After treatment discontinuation, patients were followed up for survival. Enrolled patients were divided into two cohorts: (1) a treatment-naïve cohort comprising patients who had not received any previous systemic therapy (not including somatostatin analogs), and (2) a later-line cohort comprising patients who had experienced progressive disease on or after prior systemic therapy.

Inclusion criteria in both trials were similar and included histologically or cytologically confimed diagnosis of welldifferentiated panNETs (according to World Health Organization 2000 classification) with available Ki-67 (biomarker for assessing the tumor goals) index [16], and also disease progression (per RECIST 1.0) within 12 months prior to study enrollment. Patients also had disease that was not amenable to surgery, radiation, or combined modality therapy with curative intent and Eastern Cooperative Oncology 
Group (ECOG) Performance status (PS) 0 or 1 with adequate hematologic, renal, and hepatic function.

Exclusion criteria in both trials were also similar and included: prior treatment with any TKIs or anti-vascular endothelial growth factor (VEGF) angiogenic inhibitors; diagnosis of any second malignancy within the last 5 years, except for adequately treated basal cell or squamous cell skin cancer, or in situ carcinoma of the cervix uteri; abnormal cardiac function with abnormal 12-lead electrocardiogram (ECG); and symptomatic brain metastases, spinal cord compression, or new evidence of brain or leptomeningeal disease.

Both studies were conducted in compliance with the ethical principles originating in or derived from the Declaration of Helsinki and in compliance with all International Conference on Harmonization (ICH) Good Clinical Practice (GCP) guidelines. In addition, all local regulatory requirements were followed, in particular, those affording greater protection to the safety of study participant. Written informed consent was obtained from each patient (or patient's legally authorized representative) before the patient was admitted to the study.

\subsubsection{Study Treatment}

In both trials, the starting dose of sunitinib was $37.5 \mathrm{mg}$ administered once daily orally on a CDD schedule. Patients experiencing severe toxicity could receive treatment breaks inserted into the regimen as needed. Intra-patient dose reduction to $25 \mathrm{mg}$ was permitted depending on toxicity. Intra-patient re-escalation of study medication back to a previous dose level was permitted at the discretion of the investigator upon consideration of the patient's clinical status. Dose escalation to $50 \mathrm{mg}$ daily was recommended for patients who had not yet achieved an objective disease response and who had not experienced progression or prohibitive toxicity.

\subsubsection{Study Cohorts and Outcome Assessment}

The total number of patients treated with sunitinib in each of the phase III and phase IV trials was 86 and 106, respectively, with 85 patients in the placebo arm in the phase III trial $[9,11]$. Of these, 41 and 61 treatment-naïve patients in the phase III and phase IV trials, respectively, received treatment with sunitinib and comprised the combined cohort. Treatment-naïve patients were defined as not having received any previous systemic therapies, including chemotherapy, immunotherapy, intravenous peptide receptor radiotherapy, or an investigational anticancer agent other than somatostatin analogs. The outcome data from this combined cohort of sunitinib-treated patients was compared with the treatment-naïve placebo group from the phase III trial. The data cutoffs for the phase III and phase IV trial data in the combined cohort analyses were 15 April 2009 and 19 March 2016 , respectively. For the updated OS analysis of the phase IV trial the data cutoff was 8 August 2018. Tumor response was analyzed based on investigator-assessed tumor response according to RECIST v1.0 in the phase III and phase IV trials. Additional response assessments by independent reviewers were made for treatment-naive and later-line cohorts in the phase IV trial according to RECIST [11].

\subsection{Statistical Analyses}

Kaplan-Meier methods were used to obtain estimates of PFS and OS, with corresponding two-sided 95\% CIs. The Cox proportional-hazards model was used to calculate hazard ratios; $p$ values were one-sided and based on an unstratified log-rank test [9]. For ORR, point estimates were provided as well as two-sided $95 \%$ CIs using the exact method based on the $F$ distribution; $p$ values were based on a one-sided Pearson $\chi^{2}$ test. Descriptive statistics were used to summarize other parameters.

More details of an interim endpoint analysis that helped determine the early discontinuation of the phase III trial have been previously described [9].

\section{Results}

\subsection{Patients}

Patient data for the phase III trial and the phase IV trial have been previously described $[9,11]$. Briefly, in the phase III trial, between June 2007 and April 2009, 171 patients were randomized to a study arm $(n=86$ sunitinib, $n=85$ placebo). In February 2009, the DMC assessed data on patients who had undergone randomization at that point and recommended closure of the trial based on greater toxicity and number of deaths in the placebo arm, along with PFS that favored the sunitinib arm [9]. From the sunitinib arm, 41 treatment-naïve patients were included in the study subanalyses.

The median age for patients treated with sunitinib in the phase III trial was 56 years (95\% CI 25-84), similar to the phase IV trial, where the median age was 55 years $(95 \%$ CI 30-76). Patient profiles and characteristics have been previously described and were largely similar between the two trials $[9,11]$.

In the phase IV trial, 106 patients (61 treatment-naïve and 45 previously treated) were enrolled [11]. The 61 treatment-naïve patients were included in the study subanalyses. Median (range) relative dose intensity was $99.8 \%$ (55.3-125.7) for patients in the sunitinib arm of the phase 
III trial. For the sunitinib-treated patients in the phase IV trial median relative dose intensity was $92.5 \%$ (34.2-130.9).

\subsection{Progression-Free Survival (PFS) Analysis}

The investigator-assessed median (95\% CI) PFS for all patients treated with sunitinib in the phase III trial was 11.4 (7.4-19.8) months [9]. The median PFS in the combined sunitinib cohort was 12.9 (7.4-16.7) months, which showed significant benefit compared with the median PFS in the placebo arm of the phase III trial, which was 5.7 (3.6-7.9) months (HR 0.429; 95\% CI 0.245-0.752; $p=0.001$ ) (Fig. 1). PFS was $13.2(10.9-16.7)$ months [11] for all patients in the phase IV trial. In the later-line cohort in the phase IV trial $(n=45)$ a similar median PFS of $13.0(9.2-20.4)$ months was reported, as well as a similar time-to-progression (TTP; 14.5 (95\% CI 9.2-20.4) months) compared with the phase IV treatment-naïve cohort (TTP 14.8 (95\% CI 7.5-16.8) months) [11]. Time on treatment, disease progression, and survival for individual patients in the phase IV treatmentnaïve and later-line cohorts are shown in Fig. 2.

\subsection{Overall Survival (OS) Analysis}

The updated median OS for sunitinib-treated patients in the phase IV trial was 54.1 (95\% CI 37.9-Not Reached) months. Median OS could not be accurately calculated for the combined cohort or placebo due to the high number of patients censored, which was $80(78.4 \%)$ and $29(82.9 \%)$, respectively. However, based on the available survival data, HR was 0.303 (95\% CI 0.100-0.921; $p=0.013$ ) (Fig. 3). In the combined cohort, the probability of survival at year 1 for treatment-naïve patients in the sunitinib arm was $92.0 \%$ (95\% CI 83.8-96.1) versus $86.3 \%$ (95\% CI 67.4-94.7) for treatment-naïve patients in the placebo arm (placebo arm cutoff 15 April 2009).

\subsection{Objective Response Rate (ORR) Analysis}

The ORR reported for all patients in the sunitinib arm in the phase III trial was 9.3\% (95\% CI 3.2-15.4) with unconfirmed partial responses omitted due to early study termination [9]. For the combined sunitinib cohort, investigator-assessed ORR was $16.7 \%$ (95\% CI 10.0-25.3) (Table 1 and Fig. 4). No confirmed objective responses were reported in the placebo group. In the combined sunitinib cohort, 39 patients (38.2\%) had measurable tumor shrinkage of $\leq 30 \%$, seven patients (6.9\%) had tumor shrinkage between $30 \%$ and $60 \%$, and five patients (5.0\%) had $\geq 60 \%$ tumor shrinkage (Fig. 4). In the phase IV trial, the ORR, as assessed per RECIST, was $24.5 \%$ (95\% CI 16.7-33.8) [11] for all patients. The ORR for the treatment-naïve and later-line cohorts in the phase IV trial was $21.3 \%$ (95\% CI 11.9-33.7) and 28.9 (95\% CI 16.4-44.3), respectively [11]. The ORR, as assessed by independent third-party assessment, was $21.7 \%$ (95\% CI 14.3-30.8) for all patients in the phase IV trial compared with $23.0 \%$ (95\% CI 13.2-35.5) for the treatment-naïve cohort and 20.0\% (95\% CI 9.6-34.6) for the later-line cohort.

\subsection{Safety}

The safety of sunitinib in the phase III and IV trials has been previously described. Briefly, in the sunitinib arm of the phase III trial the treatment-related AEs with the highest rate
Fig. 1 Progression-free survival (PFS) for the combined phase III and phase IV treatmentnaïve sunitinib cohort versus the treatment-naïve placebo cohort in the phase III trial. $C I$ confidence interval

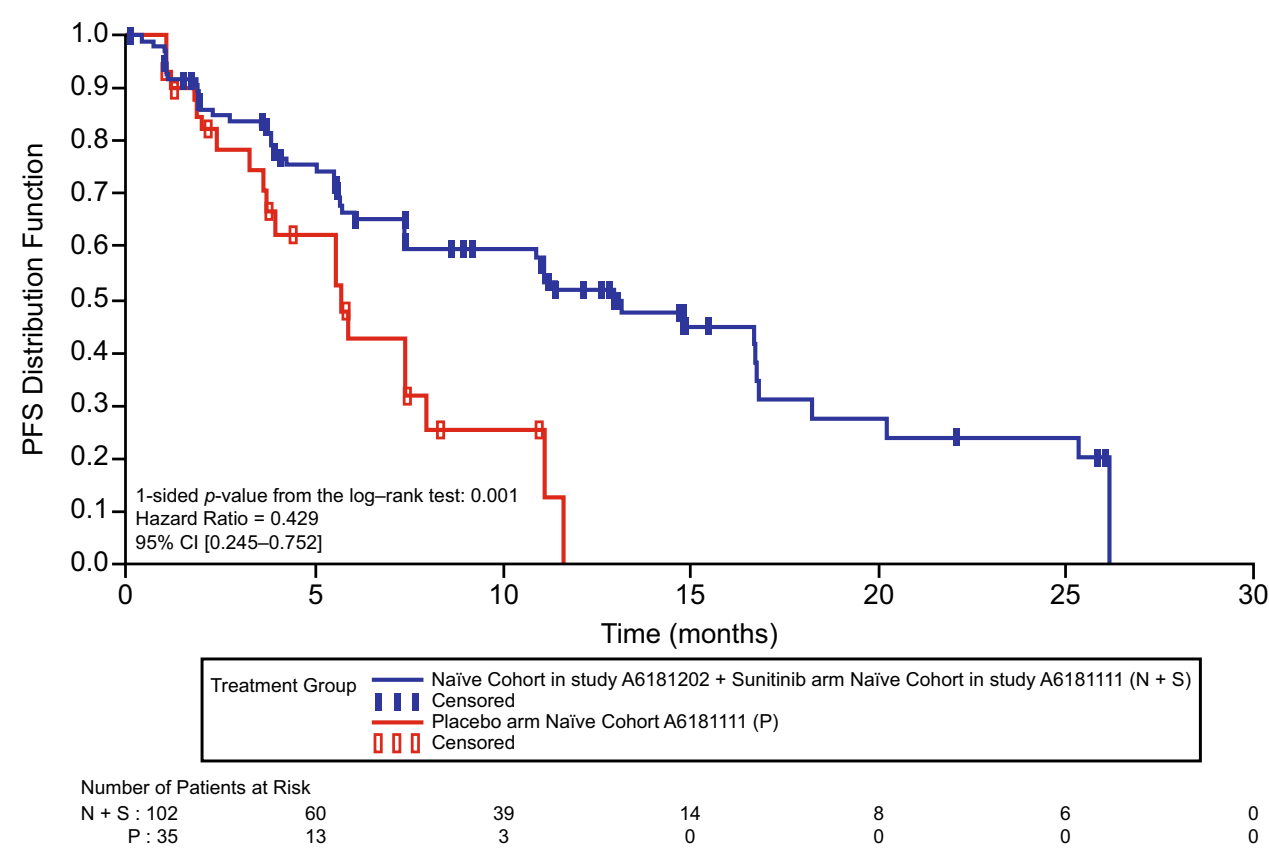


Fig. 2 Tumor swimmer plot investigator-assessed response for phase IV full analysis set. a Treatment-naïve cohort phase IV trial and $\mathbf{b}$ later-line cohort phase IV trial. $P D$ progressive disease
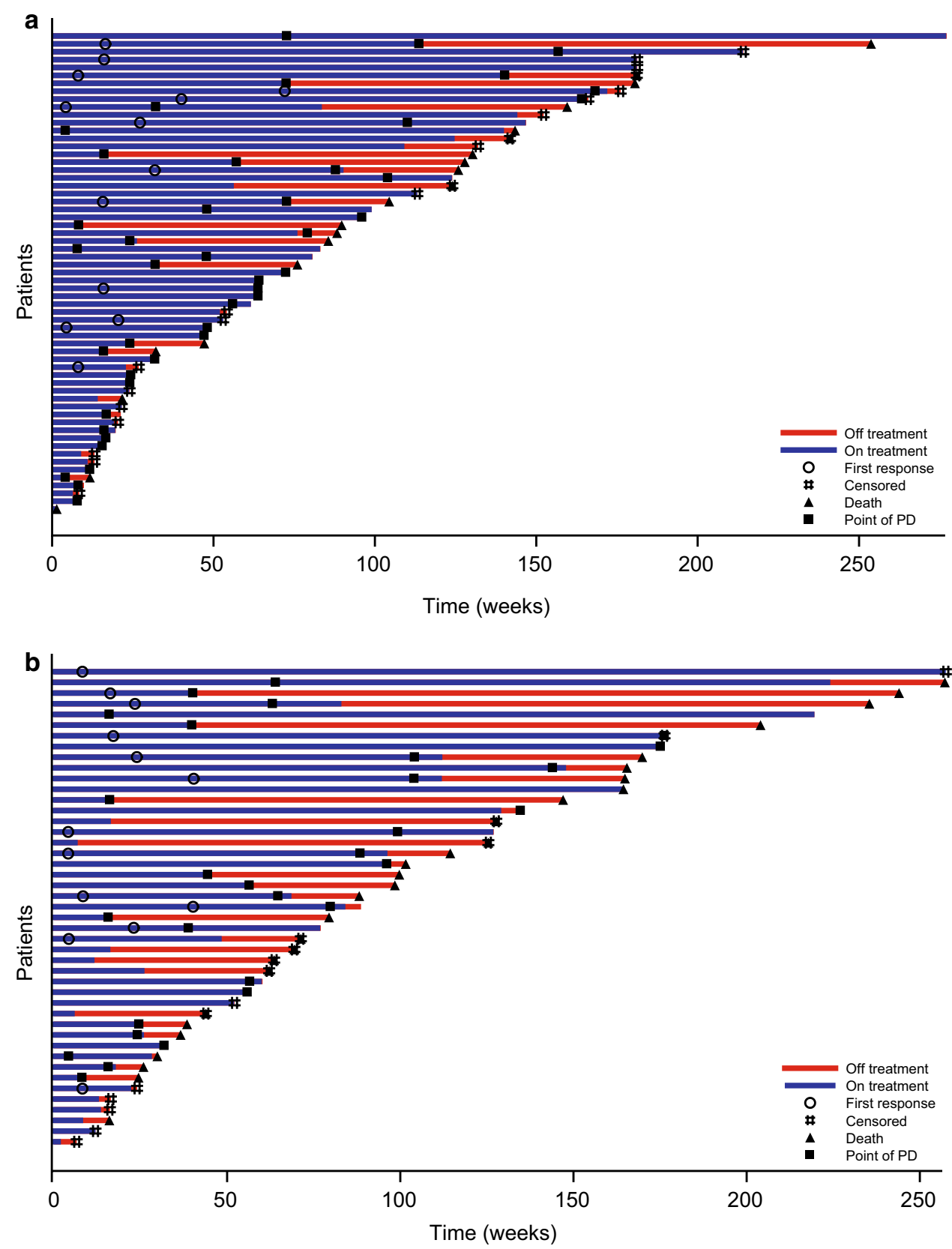

of occurrence were diarrhea (59\%), nausea (45\%), asthenia (34\%), vomiting (34\%), and fatigue (32\%), all occurring in $\geq 30 \%$ of patients. Hypertension of any grade occurred in $26 \%$ of patients in the sunitinib arm, with palmar-plantar erythrodysesthesia syndrome (PPE) occurring in 23\% [9]. In the phase IV trial the most common (i.e., occurring in $\geq 30 \%$ of patients) of any-grade treatment-related AEs were neutropenia (53.8\%), diarrhea (46.2\%), leukopenia (43.4\%), PPE (31.1\%), and thrombocytopenia (30.2\%). Hypertension occurred in $19.8 \%$ of patients [11]. Overall, most AEs were grade 1 or 2 for both trials and the general safety profile for both trials was similar to that seen previously for sunitinib $[5,7,9,11]$.
The median duration of treatment for the sunitinib arm $(n=83)$ of the phase III trial was 4.6 (95\% CI 0.4-19.8) months. Patients continued on treatment after the phase III trial was stopped and unblinded, moving to an extension study. Thus, the relatively short median treatment duration reported does not include time on treatment after the end of the trial. For patients treated with sunitinib $(n=106)$ in the phase IV trial, the median duration of treatment was 12.0 (95\% CI 0.2-63.7) months.

In the phase III trial, $31 \%$ and $11 \%$ of patients in the sunitinib and placebo arms, respectively, had at least one dose reduction, and $17 \%$ and $8 \%$ of patients who received sunitinib and placebo, respectively, discontinued due to AEs. 
Fig. 3 Overall survival for the combined phase III and phase IV treatment-naïve sunitinib cohort versus the treatmentnaïve placebo cohort in the phase III trial. $C I$ confidence interval

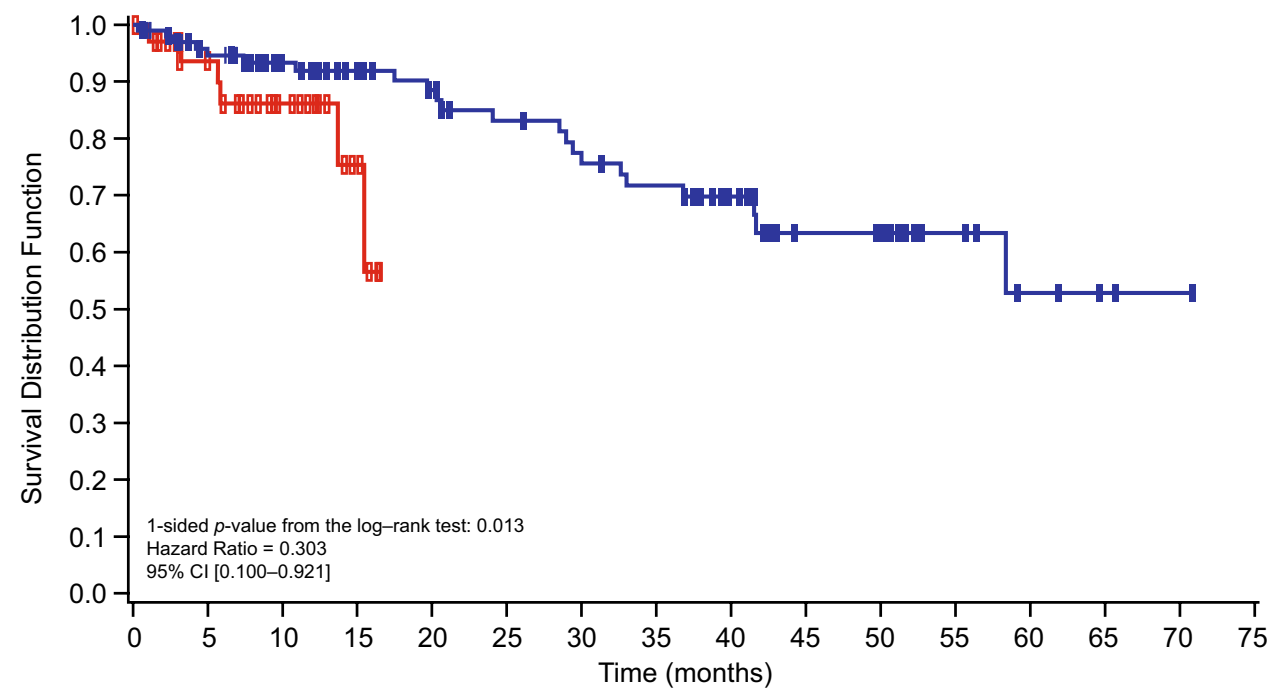

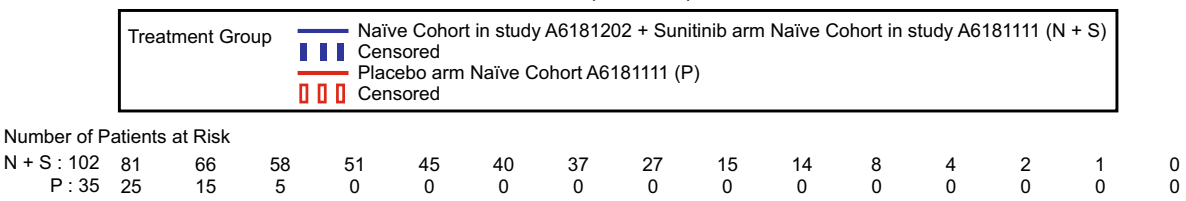

Table 1 Best overall response and objective response rate for combined phase III and phase IV treatment-naïve sunitinib cohort and placebo arm treatment-naïve cohort in the phase III trial

\begin{tabular}{lll}
\hline & $\begin{array}{l}\text { Sunitinib arm } \\
\text { cohort }(N= \\
102)\end{array}$ & $\begin{array}{l}\text { Placebo arm } \\
\text { cohort }(N= \\
35)\end{array}$ \\
\hline $\begin{array}{l}\text { Best overall response, } n(\%) \\
\text { CR }\end{array}$ & $3(2.9)$ & 0 \\
PR & $14(13.7)$ & 0 \\
SD & $65(63.7)$ & $22(62.9)$ \\
PD & $13(12.7)$ & $6(17.1)$ \\
Indeterminate & $7(6.9)$ & $7(20.0)$ \\
Objective response rate (CR + PR) & $17(16.7)$ & 0 \\
95\% exact CI & $10.0-25.3$ & $0.0-10.0$ \\
Versus placebo arm naïve cohort in $^{\mathrm{a}}$ & & \\
pivotal phase III trial & & \\
Treatment difference $^{\text {95\% CI of difference }}$ & 16.667 & \\
$p$ value $^{\mathrm{c}}$ & $9.4-23.9$ & \\
\hline
\end{tabular}

$C I$ confidence interval, $C R$ complete response, $N$ number of patients, $P D$ progressive disease, $P R$ partial response, RECIST Response Evaluation Criteria In Solid Tumors, $S D$ stable disease

${ }^{a}$ Using exact method based on binomial distribution

${ }^{\mathrm{b}}$ Calculated based on a normal distribution

${ }^{\mathrm{c}}$ One-sided $p$ value from Pearson chi-square test

The most common AE leading to discontinuation in the phase III trial in the sunitinib group was fatigue (4\%) [9]. In the phase IV trial, $9.4 \%$ of patients were discontinued due to treatment-related AEs, including $8.2 \%$ of patients in the treatment-naïve cohort and $11.1 \%$ of patients in the laterline cohort. A total of $18.9 \%$ of patients had dose reductions due to treatment-related AEs, including $24.6 \%$ and $11.1 \%$ patients in the treatment naïve and the later-line cohort, respectively. The most frequent $(\geq 5 \%) \mathrm{AE}$ leading to dose reductions was neutropenia $(6.6 \%)$.

\section{Discussion}

The combined sunitinib-treated cohort analysis of the phase III and IV trials confirmed the PFS and ORR benefit reported from the phase III placebo-controlled and phase IV trials, respectively.

Outcomes for PFS and ORR were numerically better in patients treated with sunitinib in the combined cohort versus the phase III trial, supporting the survival benefit of sunitinib reported in the phase III trial. At the time of treatment of patients in the phase IV trial, data generated from the phase III trial had been disclosed and patient management has improved since then. As a consequence, longer PFS and higher ORR in the phase IV trial may be due to better maintaining patients on treatment and managing AEs with experience and confidence in sunitinib, as has been reported for other TKIs [17]. This is further supported by the longer median duration of treatment for patients receiving sunitinib in the phase IV trial compared with the phase III trial (11.7 vs. 4.6 months, respectively) $[9,11]$. Sunitinib dose intensity did not appear to contribute to the difference between phase III and phase IV trial outcomes as it was generally similar for patients in both trials. Further, improvement of patient 
Fig. 4 Maximum percent change from baseline in the sum of the longest diameters of target lesions as assessed by the investigators. a Combined phase III and phase IV treatmentnaïve cohort sunitinib arm and $\mathbf{b}$ treatment-naïve cohort placebo arm from the phase III trial. $C R$ complete response, $N E$ not evaluable, $P D$ progressive disease, $P R$ partial response, $S D$ stable disease

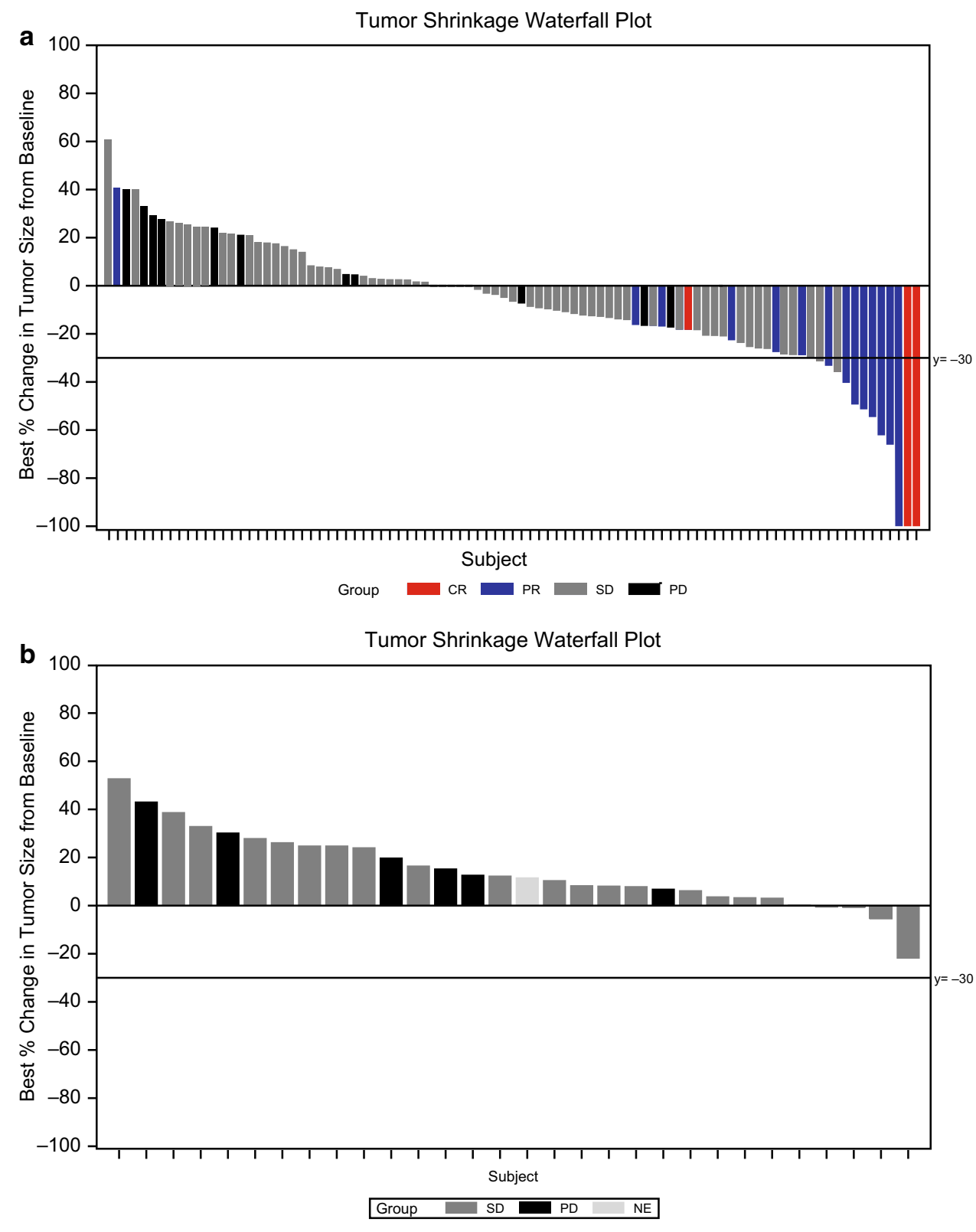

management is not captured by looking at the frequency of any-grade adverse events. The overall safety of sunitinib in both trials was generally similar to the safety profiles of sunitinib reported in other studies $[5,7,18]$.

The PFS (12.9 months) in the combined sunitinib-treated cohort with a significant benefit versus placebo (HR 0.429; 95\% CI 0.245-0.752; $p=0.001$ ) is consistent with the PFS (10 months) benefit observed in heavily pre-treated patients with panNETs in a real-world clinical setting [19]. Similarly, the ORR for the combined sunitinib-treated cohort reported in the present analysis (16.7\%) is consistent with the ORR from two studies in real-world settings in patients from Italy (17.5\%) and Japan (13.7\%) [19, 20]. Altogether, these data demonstrate the clinical benefit of sunitinib treatment in patients with panNETs, regardless of previous treatment.

The encouraging tumor response rate seen in the combined cohort in this analysis provides further support for the clinical benefit of sunitinib in panNETs, particularly in the context of the rising interest in using ORR as a primary endpoint for panNETs trials [12-14]. Compared with PFS, ORR specifically looks at tumor shrinkage and significantly higher rates of shrinkage demonstrates significant responsiveness of panNETs to sunitinib. The potential difference in ORR between the treatment-naïve and later-line cohort also suggests that ORR could be used to identify patient characteristics or treatment differences that can impact overall 
outcome but may be more easily detectable, earlier by differences in ORR.

Cross-trial comparisons should be interpreted with caution because of potential differences in trial design and patient population. However, the updated median OS for the patients treated with sunitinib in the phase IV trial (54.1 months; 95\% CI 37.9-NR) compares favorably with the median OS reported for everolimus in the follow-up of the RADIANT-3 trial, which was 44.0 months $(95 \%$ CI 35.6-51.8) [21]. The median OS could not be calculated for the combined cohort or for the phase III placebo cohort in this study.

As noted in a recently published review and meta-analysis, sunitinib may be a reasonable option for treating patients with panNETs who require tumor shrinkage, and its activity in terms of tumor response is likely underestimated with the currently utilized RECIST methods [22].

This study was limited by the large proportion of patients who were censored in the OS analysis, which prevented an accurate calculation of median OS for the combined sunitinib-treated cohort and placebo cohort from the phase III trial. Furthermore, adjudication of AEs by investigators should be regarded with caution in placebo control studies as many events related to tumor progression such as asthenia, weight loss, and abdominal pain may lead to confusion between drug-related toxicity and tumor progression. As a result, $11 \%$ of patients in the placebo group were reported to experience toxicity that were attributed to therapy.

\section{Conclusions}

The combined sunitinib cohort data from two phase III and phase IV trials are consistent with and support the PFS and ORR benefits reported in the individual trials. The ORR associated with sunitinib in these trials merits further investigation in light of increased interest in ORR as a primary endpoint in panNETs clinical trials. The benefit-risk profile of sunitinib was confirmed to be favorable for this patient population.

\section{Declarations}

Funding This study was sponsored by Pfizer. Medical writing support was provided by Vardit Dror, PhD and Charles Cheng, MS, of Engage Scientific Solutions, and funded by Pfizer.

Conflict of interest Eric Raymond is a consultant for and has received grants for clinical trials from Ipsen, Novartis, Eli Lilly, and Pfizer. Matthew H. Kulke has participated on advisory boards for Ipsen and Novartis. Nicola Fazio has received honoraria from Pfizer; he has participated on advisory boards for Novartis, Pfizer, and Ipsen, Merck Serono, MSD, and AAA; and his institution has received research funds from Novartis and Merck Serono. Brad Rosbrook and Kathrine Fernandez are employees of and hold stock in Pfizer.
Ethics approval Both studies were conducted in compliance with the ethical principles originating in or derived from the Declaration of Helsinki and in compliance with all International Conference on Harmonization (ICH) Good Clinical Practice (GCP) guidelines. In addition, all local regulatory requirements were followed, in particular those affording greater protection to the safety of study participant.

Consent to participate Written informed consent was obtained from each patient (or the patient's legally authorized representative) before the patient was admitted to the study.

Consent for publication Not applicable.

Availability of data and material Upon request, and subject to certain criteria, conditions, and exceptions (see https://www.pfizer.com/scien ce/clinical-trials/trial-data-and-results for more information), Pfizer will provide access to individual de-identified participant data from Pfizer-sponsored global interventional clinical studies conducted for medicines, vaccines and medical devices (1) for indications that have been approved in the USA and/or EU or (2) in programs that have been terminated (i.e., development for all indications has been discontinued). Pfizer will also consider requests for the protocol, data dictionary, and statistical analysis plan. Data may be requested from Pfizer trials 24 months after study completion. The de-identified participant data will be made available to researchers whose proposals meet the research criteria and other conditions, and for which an exception does not apply, via a secure portal. To gain access, data requestors must enter into a data access agreement with Pfizer.

Code availability Not applicable.

Authors' contributions All authors were involved in the study conception/design. Material preparation and analysis were performed by BR. All authors were involved in the interpretation of data. All authors contributed to the drafting of the manuscript and approved the final version.

Open Access This article is licensed under a Creative Commons Attribution-NonCommercial 4.0 International License, which permits any non-commercial use, sharing, adaptation, distribution and reproduction in any medium or format, as long as you give appropriate credit to the original author(s) and the source, provide a link to the Creative Commons licence, and indicate if changes were made. The images or other third party material in this article are included in the article's Creative Commons licence, unless indicated otherwise in a credit line to the material. If material is not included in the article's Creative Commons licence and your intended use is not permitted by statutory regulation or exceeds the permitted use, you will need to obtain permission directly from the copyright holder. To view a copy of this licence, visit http://creativecommons.org/licenses/by-nc/4.0/.

\section{References}

1. Sharma J, Duque M, Saif MW. Emerging therapies and latest development in the treatment of unresectable pancreatic neuroendocrine tumors: an update for clinicians. Therap Adv Gastroenterol. 2013;6:474-90.

2. Casanovas O, Hicklin DJ, Bergers G, Hanahan D. Drug resistance by evasion of antiangiogenic targeting of VEGF signaling in latestage pancreatic islet tumors. Cancer Cell. 2005;8:299-309.

3. Fjallskog ML, Hessman O, Eriksson B, Janson ET. Upregulated expression of PDGF receptor beta in endocrine pancreatic tumors 
and metastases compared to normal endocrine pancreas. Acta Oncol. 2007;46:741-6.

4. Faivre S, Demetri G, Sargent W, Raymond E. Molecular basis for sunitinib efficacy and future clinical development. Nat Rev Drug Discov. 2007;6:734-45.

5. Motzer RJ, Hutson TE, Tomczak P, Michaelson MD, Bukowski RM, Rixe O, et al. Sunitinib versus interferon Alfa in metastatic renal-cell carcinoma. N Engl J Med. 2007;356:115-24.

6. Raymond E, Hammel P, Dreyer C, Maatescu C, Hentic O, Ruszniewski $\mathrm{P}$, et al. Sunitinib in pancreatic neuroendocrine tumors. Target Oncol. 2012;7:117-25.

7. Pfizer Inc. Sutent (sunitinib) prescribing information. Pfizer Inc, New York, NY, USA. 2018. http://labeling.pfizer.com/ShowLabeli ng.aspx?id=607. Accessed 20 Dec 2019.

8. Blumenthal GM, Cortazar P, Zhang JJ, Tang S, Sridhara R, Murgo A, et al. FDA approval summary: sunitinib for the treatment of progressive well-differentiated locally advanced or metastatic pancreatic neuroendocrine tumors. Oncologist. 2012;17:1108-13.

9. Raymond E, Dahan L, Raoul JL, Bang YJ, Borbath I, LombardBohas C, et al. Sunitinib malate for the treatment of pancreatic neuroendocrine tumors. N Engl J Med. 2011;364:501-13.

10. Faivre S, Niccoli P, Castellano D, Valle JW, Hammel P, Raoul $\mathrm{JL}$, et al. Sunitinib in pancreatic neuroendocrine tumors: updated progression-free survival and final overall survival from a phase III randomized study. Ann Oncol. 2017;28:339-43.

11. Raymond E, Kulke MH, Qin S, Yu X, Schenker M, Cubillo A, et al. Efficacy and safety of sunitinib in patients with well-differentiated pancreatic neuroendocrine tumours. Neuroendocrinology. 2018;107:237-45.

12. Grande E, Lopez C, Alonso-Gordoa T, Benavent M, Capdevila J, Teule A, et al. The SUNEVO (GETNE-1408) trial to evaluate the activity and safety of the combination of sunitinib with evofosfamide (TH-302) in patients with G1/G2 metastatic pancreatic neuroendocrine tumours (pNETs) naïve forsystemic treatment: A phase II study of the Spanish Task Force Group for Neuroendocrine and Endocrine Tumors (GETNE). J Clin Oncol. 2019;37(15_suppl):4105.

13. Price TJ, Cehic G, Wachter EA, Kirkwood I, Sebbon R, Leopardi $\mathrm{L}$, et al. A phase I study of oncolytic immunotherapy of metastatic neuroendocrine tumors using intralesional rose bengal disodium: cohort 1 results. J Clin Oncol. 2019;37(15_suppl):4102.

14. Al-Toubah TE, Morse B, Strosberg JR. Retrospective study of capecitabine and temozolomide in advanced lung neuroendocrine neoplasms. J Clin Oncol. 2019;37(15_suppl):8542.

15. Falconi M, Plockinger U, Kwekkeboom DJ, Manfredi R, Korner $\mathrm{M}$, Kvols L, et al. Well-differentiated pancreatic nonfunctioning tumors/carcinoma. Neuroendocrinology. 2006;84:196-211.

16. Childs A, Kirkwood A, Edeline J, Luong TV, Watkins J, Lamarca A, et al. Ki-67 index and response to chemotherapy in patients with neuroendocrine tumours. Endocr Relat Cancer. 2016;23:563-70.

17. Faivre S, de Gramont A, Raymond E. Learning from 7 years of experience with sorafenib in advanced HCC: sorafenib better than sorafenib? Target Oncol. 2016;11:565-7.

18. Ravaud A, Motzer RJ, Pandha HS, George DJ, Pantuck AJ, Patel A, et al. Adjuvant sunitinib in high-risk renal-cell carcinoma after nephrectomy. N Engl J Med. 2016;375:2246-54.

19. Rinzivillo M, Fazio N, Pusceddu S, Spallanzani A, Ibrahim T, Campana D, et al. Sunitinib in patients with pre-treated pancreatic neuroendocrine tumors: a real-world study. Pancreatology. 2018;18:198-203.

20. Sato K, Toyoshima Y, Moriyama S, Endo Y, Ito T, Ohki E. Realworld use of sunitinib in Japanese patients with pancreatic neuroendocrine tumors: results from a post-marketing surveillance study. Cancer Chemother Pharmacol. 2019;83:201-7.

21. Yao JC, Pavel M, Lombard-Bohas C, Van Cutsem E, Voi M, Brandt U, et al. Everolimus for the treatment of advanced pancreatic neuroendocrine tumors: overall survival and circulating biomarkers from the randomized, phase III RADIANT-3 study. J Clin Oncol. 2016;34:3906-13.

22. Pozzari M, Maisonneuve P, Spada F, Berruti A, Amoroso V, Cella $\mathrm{CA}$, et al. Systemic therapies in patients with advanced well-differentiated pancreatic neuroendocrine tumors (PanNETs): When cytoreduction is the aim. A critical review with meta-analysis. Cancer Treat Rev. 2018;71:39-46. 